\title{
Solitary wave propagation in antiferroelectric liquid crystal cells and the quadrupolar term in the interlayer interaction
}

\author{
Jang-Kun Song, Atsuo Fukuda, and J. K. Vij* \\ Department of Electronic and Electrical Engineering, Trinity College, University of Dublin, Dublin 2, Ireland \\ (Received 30 November 2006; revised manuscript received 10 May 2007; published 20 July 2007) \\ Planar antiferroelectric liquid crystal (AFLC) cells exhibit propagating solitary waves. By measuring the \\ retardation and the apparent tilt angle, we find that the conventional model for the solitary wave propagation in \\ AFLCs is not generally applicable. The model being proposed here shows that the solitary wave propagation \\ both along and normal to the smectic layer plane can be explained by a double well potential energy governing \\ the relative orientations of the $\mathbf{c}$ directors in the adjacent smectic $C$ layers with an energy barrier between the \\ synclinic and the anticlinic states. The quadrupolar term of the interlayer interaction is found to be significantly \\ large in a pure AFLC.
}

DOI: 10.1103/PhysRevE.76.011708

PACS number(s): 61.30.Gd, 42.70.Df, 77.84.Nh

Antiferroelectric liquid crystals (AFLCs) have attracted many researchers' interest due not only to scientific but also for display applications [1-10]. Especially, the field driven phase transition from antiferroelectric (AF) state to ferroelectric $(F)$ state, that is, the switching of AFLC cells has been studied in detail [3-8] for application purpose. So, the switching of AFLC cells shows various interesting phenomena (threshold, hysteresis, fast response, continuous grayscale, etc.) useful in applications.

The solitary wave propagation $[11,12]$, which is a consecutive transition of the anticlinic orders to the synclinic orders occurring as dominoes, is one of the most crucial aspects of the switching in AFLC cells. As shown in the microscopic images in Fig. 1, the solitary wave propagation is easily observed in the antiferroelectric cells as propagating boundaries of finger domains. A domain propagation observed in a surface-stabilized ferroelectric liquid crystal cell is induced by the special cell structure [13]. However, this propagation in a AFLC cell is mainly an intrinsic property of the phase. Hence, the physics in both cases is quite different in spite of the similarity of the behavior. So far, the solitary wave phenomenon has been studied mainly for understanding the switching properties of AFLC cells from the viewpoint of applications $[5,6]$. However, the solitary wave itself has rarely been studied $[1,14]$, and even its mechanism is not clear. This is the starting point of this paper.

In order to have the solitary wave, there should exist two energy minima in the two states ( $\mathrm{AF}$ and $\mathrm{F}$ states) and a energy barrier between the two states must also exist $[11,12]$. Li et al. [1] observed and characterized the propagating solitary waves in a AFLC cell. They assumed that the necessary condition for the generation of the solitary wave is to have the negative dielectric anisotropy, which is the mechanism to induce a synclinic local minimum in the interlayer potential energy. The initial state of the cells was also assumed such that the $\mathbf{c}$ directors of the even layers lie at $\phi=0$, which are not affected by the applied voltage, while those of the odd layers are at $\phi=\pi$ and they transfer to the position of the

\footnotetext{
*Author to whom correspondence should be addressed. jvij@tcd.ie
}

even layers by the external field. In this definition, $\phi$ is the angle between the directors and $x$ axis. The latter lies along the glass plate. Thus, in their model, the c directors are horizontally aligned (HAF) being perpendicular to the applied field. However, it has been reported experimentally $[2,15-17]$ and proven theoretically $[7,15]$ that the virgin chiral AF stage is uncoiled into the unwound AF state by a relatively low field, where the c directors are aligned vertically (VAF), that is, parallel to the field, and it turns into the F state at a sufficiently large field. This contradicts the basic assumption made by $\mathrm{Li}$ et al. [1]. Whether the unwound AF state is a VAF or a HAF state is an important starting point for the solitary wave propagation to occur. If the unwound
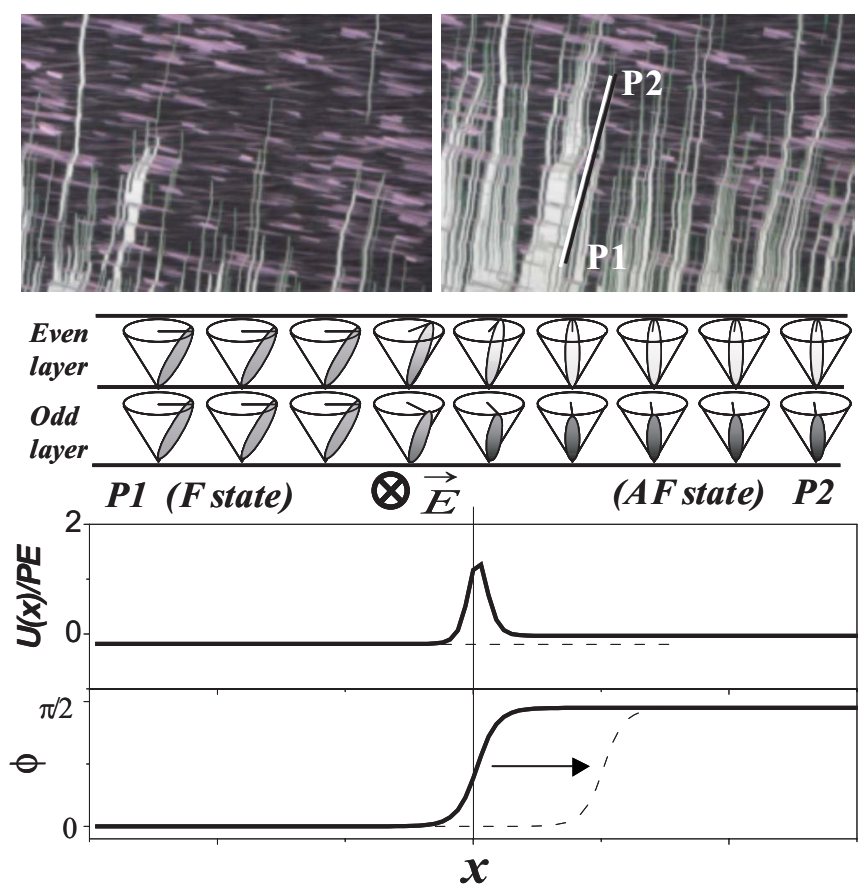

FIG. 1. (Color online) Microscopic images of the solitary waves in a planar AFLC cell using MC881 (upper), director distributions near the boundary for the two states P1 and P2 (middle), and the potential energy $U$ and the angle $\phi$ calculated from Eqs. (1) and (8) (lower). 

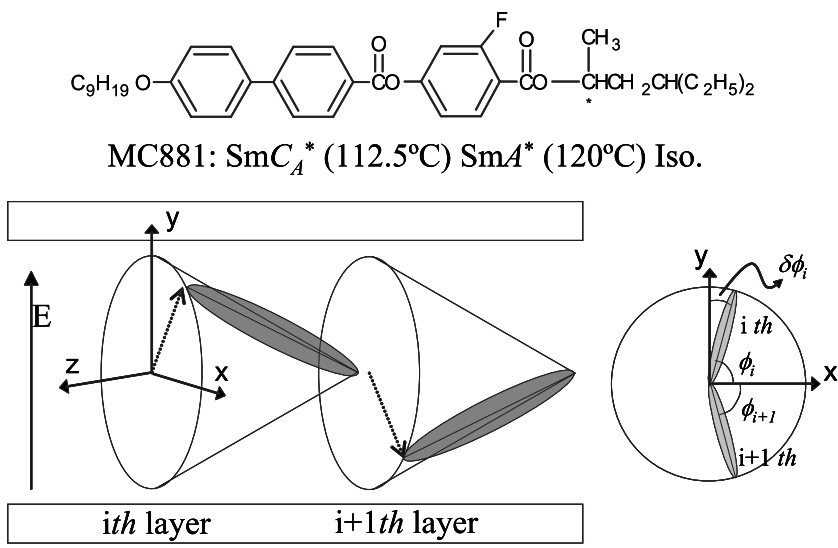

FIG. 2. Chemical structure of MC881. Molecular and $\mathbf{c}$ directors in the intermediate unwound antiferroelectric state (VAF state) in a planar AFLC cell.

AF state is VAF state as already found [15], the solitary wave propagation cannot appear unless their model is altered, as shown here.

We used MC881 for the electro-optic measurements, which is an optically pure AFLC compound synthesized by Mitsubishi Gas Chemical Company Inc. (MGC) (Fig. 2). This material does not have $\mathrm{Sm} C^{*}$ phase. The phase sequence is Iso. $\left(120^{\circ} \mathrm{C}\right) \mathrm{Sm} A\left(112.5^{\circ} \mathrm{C}\right) \mathrm{Sm}_{A}{ }^{*}$. To check the direction of the $\mathbf{c}$ director in the intermediate unwound state, we measured the retardation by increasing the field using a PEM system [18] which enables a measurement of the difference in the refractive indices along the two eigen axes in the plane parallel to the cell. In the lower part of Fig. 3 , the increasing retardation at low voltages indicates that the c directors lie close to $y$ axis, which is the VAF state shown in Fig. 2. The HAF state has a lower retardation than the virgin chiral antiferroelectric state. The increasing apparent tilt angle at low fields in Fig. 2 also leads to the same conclusion. The sudden jumps in the retardation and tilt angle indicate the AF-F transition.

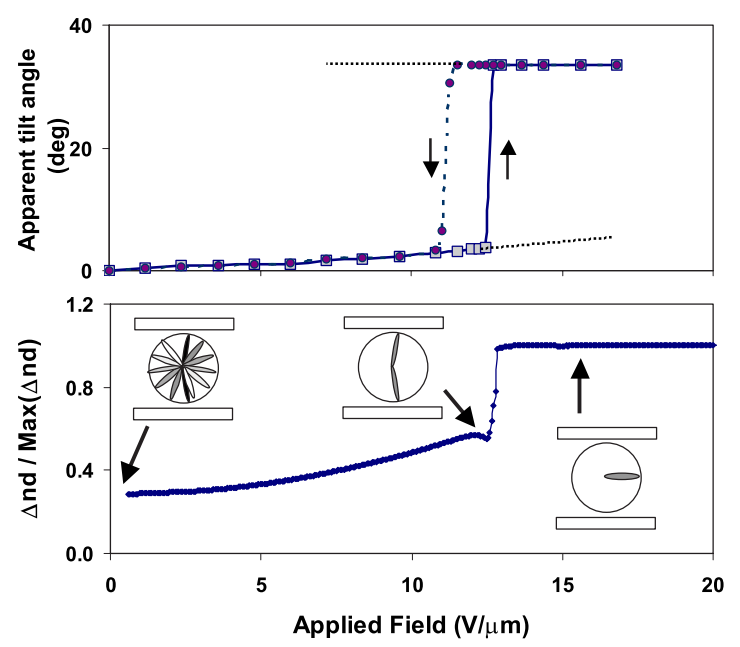

FIG. 3. (Color online) Apparent tilt angle (upper, $25 \mu \mathrm{m}$ planar cell) and retardation $\Delta n d$ (lower, $5 \mu \mathrm{m}$, planar cell) as a function of the applied field.
To model the solitary wave in the VAF state, we start off with the free energy expression of an unwound helical limiting case. The free energy of $i$ th layer can be written using the coordinate system and definitions of angles in Fig. 2 as

$$
\begin{aligned}
f_{i}= & \frac{K}{2} \sin ^{2} \theta\left(\frac{\partial \phi_{i}(x)}{\partial x}\right)^{2}-P_{0} E \cos \phi_{i}(x) \\
& -\frac{\Delta \epsilon^{\prime}}{8 \pi} \sin ^{2} \phi_{i}(x) E^{2}+V[\Delta \phi(x)] \\
\equiv & \frac{K}{2} \sin ^{2} \theta\left(\frac{\partial \phi_{i}(x)}{\partial x}\right)^{2}+U\left[\phi_{i}(x), \Delta \phi(x)\right] .
\end{aligned}
$$

Here, $V(\Delta \phi)$ is the interlayer interaction depending on the angle between the adjacent layers, and the effective dielectric anisotropy $\Delta \epsilon^{\prime}=\Delta \epsilon \sin ^{2} \theta$. In Eq. (1), the first term of the equation is the elastic energy of the solitary wave, and the second and the third terms correspond to the coupling of the spontaneous polarization and the dielectric anisotropy with the applied field. The total potential energy $U\left[\phi_{i}(x), \Delta \phi(x)\right]$ is defined as the sum of the interlayer interaction and the coupling with the applied field.

Corresponding to Eq. (1), the equation of motion of the solitary wave can be written as $[1,11,12]$

$$
I \frac{\partial^{2} \phi}{\partial t^{2}}+\gamma \frac{\partial \phi}{\partial t}-K^{\prime} \frac{\partial^{2} \phi}{\partial x^{2}}=-\frac{d U\left(\phi_{i}, \Delta \phi\right)}{d \phi_{i}},
$$

where $I$ is the moment of inertia of a molecule and $\gamma$ is the viscosity of the medium and $K^{\prime}=K \sin ^{2} \theta$. I can be neglected in the overdamped limit where $\gamma$ is large, as is usually the case.

If only the dipolar interlayer interaction $\left\{V=V_{0}\left[\cos \left(\phi_{i}\right.\right.\right.$ $\left.\left.\left.-\phi_{i-1}\right)+\cos \left(\phi_{i+1}-\phi_{i}\right)\right]=2 V_{0} \cos (2 \phi)\right\}$ is considered as already assumed by $\mathrm{Li}$ et al. [1], the only possible solitary wave solution of Eq. (2) can be found to be

$$
\phi=2 \tan ^{-1} e^{d_{1}\left(x-v_{1} t\right)},
$$

where $\phi \equiv \phi_{i}(x)$ and

$$
\begin{gathered}
d_{1}=\left[-2\left(\frac{\Delta \epsilon^{\prime}}{8 \pi} E^{2}+2 V_{0}\right) / K^{\prime}\right]^{1 / 2}, \\
v_{1}=\frac{P E}{\gamma d_{1}} .
\end{gathered}
$$

This solution does not correspond to the solitary wave for the AF-F transition usually observed in the AF cells, but does that of the transition from one $\mathrm{F}$ state to another $\mathrm{F}$ state at very large fields. Note that in the solitary wave solution [Eq. (3)], directors change from $\phi=0$ (for $x=-\infty$ ) to $\phi=\pi$ (for $x=+\infty)$, that is, from $\Delta \phi=0$ to $\Delta \phi=2 \pi$. To have a real value of $d_{1}$, the dielectric anisotropy should be negative and the applied field $E>4 \sqrt{-\pi V_{0} / \Delta \epsilon^{\prime}}\left(\sim 1.6 \times 10^{3}\right.$ stat $\left.\mathrm{V} / \mathrm{cm}^{-1}\right)$ $\left(>E_{\mathrm{th}}\right)$, which is quite a large field. Even though this solitary wave is physically possible, the moving speed is too fast to be observed in the usual microscopic experiment. Thus, with only the dipolar term present in the interlayer interaction, the solitary wave from the AF to F state cannot be attained irrespective of the sign of the dielectric anisotropy. This is due to 
the fact that the potential energy $U$ is not a double well potential having the synclinic and the anticlinic minima.

Now, we need to add the quadrupolar term in the interlayer interaction. The quadrupolar term was introduced in a phenomenological model of Landau expansion for AFLC by Orihara and Ishibashi [9], and an experimental evidence was suggested by Qian and Taylor [7] by observing the switching behavior in a AFLC cell. Thus, it is reasonable to add the quadrupolar term in the interlayer interactions:

$$
\begin{aligned}
V & =V_{0}(1+\cos \Delta \phi)+V_{0} b(1-\cos 2 \Delta \phi) \\
& =V_{0}\left(1+\cos 2 \phi_{i}\right)+V_{0} b\left(1-\cos 4 \phi_{i}\right) .
\end{aligned}
$$

Here, the quadrupolar coefficient $b$ is related to the relative depth of the synclinic minimum compared with that of the anticlinic minimum. The equation of motion [Eq. (2)] can be rewritten as

$$
\begin{aligned}
\gamma \frac{\partial \phi}{\partial t}-K^{\prime} \frac{\partial^{2} \phi}{\partial x^{2}}= & -P E \sin \phi+\left(\frac{\Delta \epsilon^{\prime}}{8 \pi} E^{2}+2 V_{0}\right) \sin 2 \phi \\
& -4 V_{0} b \sin 4 \phi
\end{aligned}
$$

Here, $I$ is ignored. Unfortunately, it is not easy to find the exact solution of this differential equation, but is obtained as an approximate solution of Eq. (7) as

$$
\phi(x) \approx \frac{2}{\pi} \phi_{0} \tan ^{-1} e^{d(x-v t)},
$$

where $\phi_{0}$ depends on the applied field $E$ and is the angle $\phi$ for $x \rightarrow \infty$, where the potential energy $U$ has a minimum. Equation (8) is plotted in the lower part of Fig. 1, where the c directors are highly distorted at the solitary wave and produces a large potential barrier $U$ at the same position. The large energy barrier sustains the strong elastic restoring force by the distorted $\mathbf{c}$ directors. With a slight difference between the potential energies in F and AF states, the solitary wall moves and the one state enlarges with a consequential reduction in the size of the other state. Equation (8) is the solution for the $i$ th layer, and the adjacent layer has an opposite sign of the angle of the c directors as seen in Figs. 1 and 2, so $\phi_{\text {odd }}(x)=-\phi_{\text {even }}(x)$. Here, we can find that the solitary wave in a layer is coupled with that of the adjacent layer, i.e., the solitary wave could propagate normal to the layer plane, though the speed is much slower than that along the $x$ direction. On the other hand, in the conventional model [1], only the odd layers are supposed to have the solitary waves, and the even layers stay and act as obstacles to prevent the solitary wave propagation occurring normal to the layers, and as a consequence, the solitary wave in each layer is independent of the others. Usually, the solitary wave has a fingerlike shape and builds up as a bundle of rays, the width of which is more than several thousand times the thickness of a single layer and becomes thicker with time. The special shape of the solitary wave can be explained by the proposed model as a coupling between the solitary waves in the adjacent layers.

The necessary condition for the differential Eq. (7) to have a real solution can be found by analyzing the total potential energy $U$. To have the solitary wave, $\partial U / \partial \phi=0$ and $\partial^{2} U / \partial \phi^{2}>0$ should be satisfied at $\phi=\phi_{0}$ (anticlinic mini-

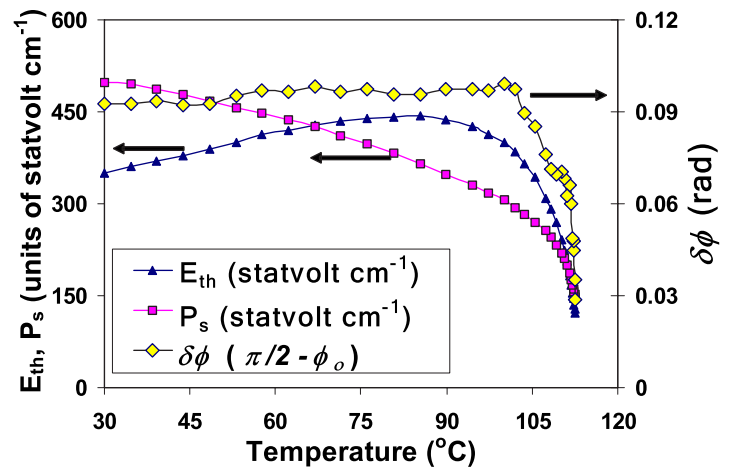

FIG. 4. (Color online) Measured $P_{s}, E_{\mathrm{th}}$, and $\delta \phi$ at $E=E_{\mathrm{th}}$ (25 $\mu \mathrm{m}$ planar cell).

mum) and 0 (synclinic minimum). This is the same condition for having the first order transition between the $\mathrm{AF}$ and the field induced F states, and the details were already studied by Qian and Taylor [7]. By ignoring the dielectric anisotropic term, we can find that when $b>1 / 20$, the potential energy $U$ has a double well potential near the critical field $\left(E_{\mathrm{th}}\right)$ and the cell could exhibit a solitary wave, and when $b>1 / 4$, the potential energy $U$ has a double well form even at the zero field. Thus, the solitary wave does not originate from the dielectric anisotropy, but does from the quadrupolar term of the interlayer interaction.

From the condition $\partial U / \partial \phi=0$ at $\phi=\phi_{0}$, we can find $\delta \phi\left(=\pi / 2-\phi_{0}\right) \ll 1$ :

$$
\delta \phi \approx \frac{P E}{4 V_{0}(4 b+1)+\frac{\Delta \epsilon^{\prime}}{4 \pi} E^{2}} .
$$

Since $\frac{\Delta \epsilon^{\prime}}{4 \pi} E^{2}$ is lower than $4 V_{0}(4 b+1), \delta \phi$ related to the apparent tilt angle increases linearly with the applied field according to Eq. (9), which accords well with the experimental results shown in Fig. 3 (see also [10]).

When the applied field $=E_{\mathrm{th}}$, where the solitary wave velocity is zero, the potential energy at the two minima (synclinic and anticlinic) should be equal to each other.

$$
U\left(E=E_{\mathrm{th}}, \phi=0\right)=U\left(E=E_{\mathrm{th}}, \phi=\phi_{0}^{\prime}\right) .
$$

By using $\partial U / \partial \phi=0$ at the two minima and Eq. (10), we can find $b, V_{0}$, and $E_{\mathrm{th}}$ :

$$
\begin{gathered}
V_{0}=P E_{\mathrm{th}} \frac{3 \cos \phi_{0}^{\prime}+2}{4\left(\cos \phi_{0}^{\prime}+1\right)^{2}}-\frac{\Delta \epsilon^{\prime}}{16 \pi} E_{\mathrm{th}}^{2}, \\
b=\frac{P E_{\mathrm{th}}}{16 V_{0} \cos \phi_{0}^{\prime}\left(\cos \phi_{0}^{\prime}+1\right)^{2}}, \\
E_{\mathrm{th}}=\frac{16 V_{0} b \cos \phi_{0}^{\prime}\left(\cos \phi_{0}^{\prime}+1\right)^{2}}{P},
\end{gathered}
$$

where $\phi_{0}^{\prime}=\phi_{0}\left(E=E_{\mathrm{th}}\right) . \quad \phi_{0}^{\prime} \quad$ can be obtained by $\phi_{0}^{\prime}$ $=\cos ^{-1}\left(\tan \theta^{\prime} / \tan \theta\right)$, where $\theta^{\prime}$ is the apparent tilt angle at $E_{\text {th }}$ and $\theta$ is the tilt angle of the director from the layer normal. 

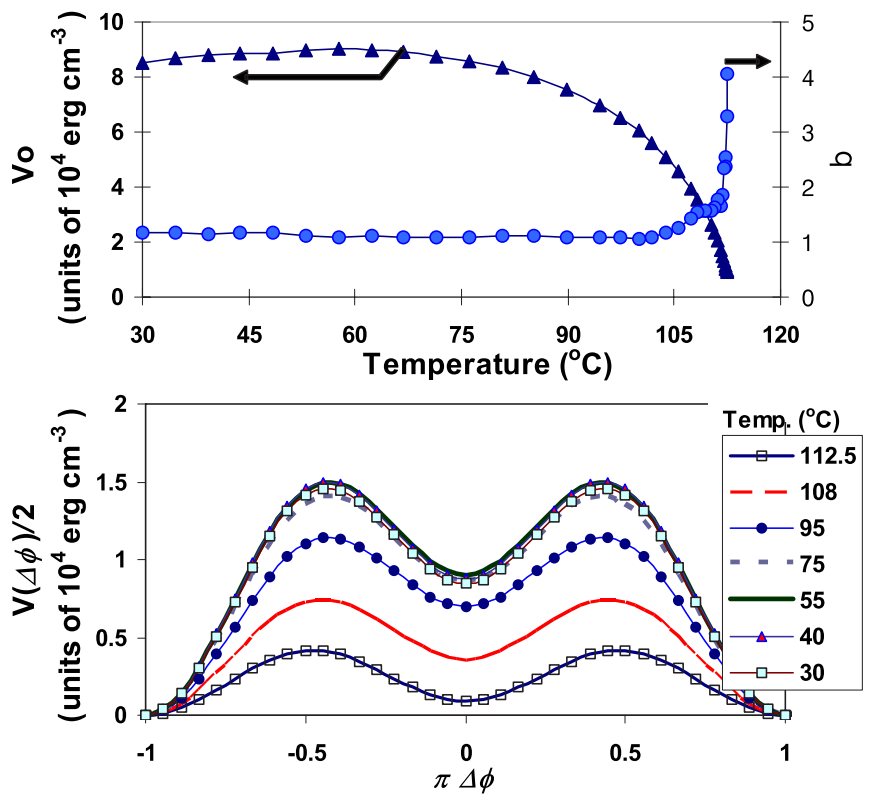

FIG. 5. (Color online) Calculated $V_{0}$ and $b$ (upper graph) and interlayer interaction energy as a function of $\Delta \phi$ (lower graph). The scale on the abscissa is multiplied by $\pi$.

Figure 4 shows the measured values of $P_{S}, E_{\mathrm{th}}$, and $\delta \phi\left(E=E_{\mathrm{th}}\right)$ using a planar cell of $25 \mu \mathrm{m}$ thickness. Using both homeotropically and planar-aligned cells, we measured $\Delta \epsilon \simeq-0.7$ in $\mathrm{SmC}_{A}{ }^{*}$ phase. The upper graph in Fig. 5 gives calculated $V_{0}$ and $b$ from the measured results. It shows that $V_{0}$ increases with decreasing temperature and is saturated at low temperatures. Meanwhile, the quadrupole coefficient $b$ is almost constant over a wide range of temperatures and increases dramatically near the transition temperature to $\operatorname{Sm} A$. This means that the synclinic minimum gets closer to the anticlinic minimum as the temperature approaches the transition temperature even though the barrier potential between them remains reasonably high as shown in the lower part of Fig. 5.

By inserting the approximate solution (8) into the differential Eq. (7) and using the measured values of $V_{0}, b$, we can find the velocity $(v)$ and the width parameter $(d)$ of the solitary wave as function of the applied field as shown in Fig. 6. Here, $v$ and $d$ are determined by fitting two equations in both sides of Eq. (7), and the coefficient of determination, $R^{2}$ for the fitting at each electric field is higher than 0.999, which means that Eq. (8) is quite an accurate approximation for the solution of the differential equation. In this simulation, we assumed that $K=10^{-6}$ dyn and $\gamma=5.0 \mathrm{P}$ so as to match the simulation results with the experimental results. The velocity of the solitary wave shows a linear dependence as function of the applied field near $E_{\mathrm{th}}$.

To summarize, the conventional model [1] explains well the basic mechanism for the solitary wave propagation in the AFLC cells, but due to its oversimplified nature, it gives

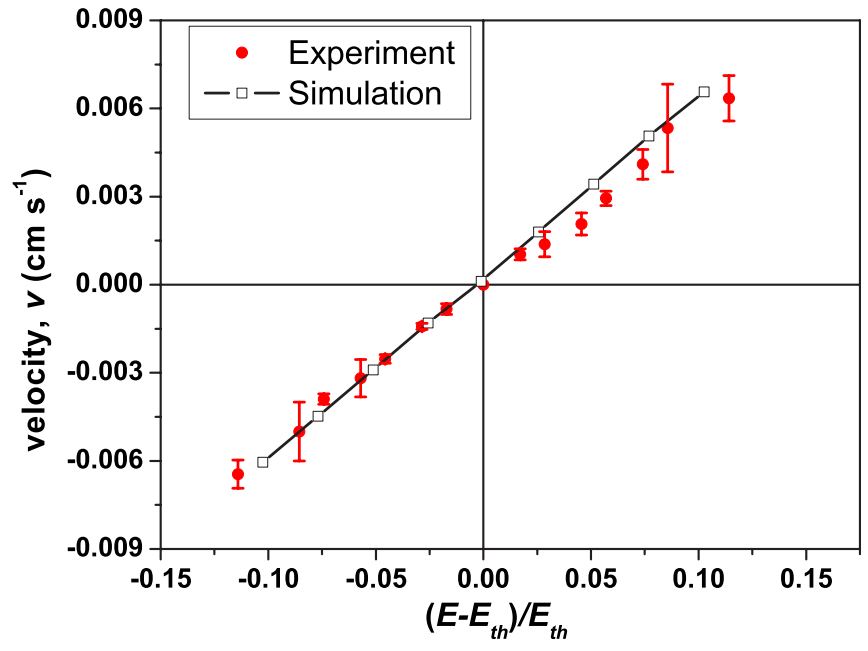

FIG. 6. (Color online) Measured velocity of the solitary wave and simulation result by using Eqs. (7) and (8) (25 $\mu \mathrm{m}$ planar cell, $50{ }^{\circ} \mathrm{C}$ ). Simulation parameter: $\gamma=5 \mathrm{P}, K=10^{-6}$ dyn. Error bars denote the standard deviation over ten measurements for each data point.

several incorrect conclusions: the solitary wave is due to the negative dielectric anisotropy, and the pretransitional effect cannot appear, and the solitary wave in each layer is separated from the neighboring ones. Our model based on the transition from the VAF to the unwound ferroelectric state can reasonably explain all of the observations: the solitary wave is due to the quadrupolar term in the interlayer interaction but is not related with the sign of the dielectric anisotropy, and the pretransitional effect always exists, and the solitary wave of each layer affects the neighboring layers and can also propagate normal to the layers. The solitary wave builds up like a bundle of rays giving it the shape of a finger as observed. We predict the generation of a new type of solitary wave due to a negative dielectric anisotropy when a very large square shaped field is applied, even though its detection may not be easy. We calculate $V_{0}$, and the quadrupolar coefficient, $b$, of the interlayer interaction in a pure AFLC in the entire range of temperature of the $\mathrm{SmC}_{A}^{*}$ phase by observing the solitary wave. Surprisingly, the results show that even the pure AFLC not having $\mathrm{SmC}^{*}$ phase has a deep synclinic minimum since the quadrupolar coefficient $b$ $\simeq 1.14$ is larger than that for a commercial AFLC mixture (Chisso, CS4001: $b \simeq 0.67$ ) having $\mathrm{Sm} C^{*}$ phase [8]. Moreover $b$ increases exponentially near the transition. This means that the antiferroelectric coupling becomes weaker near the transition temperature to the $\operatorname{Sm} A$ phase.

Work was funded by SFI(02/IN.1/I.031). J.K.Song thanks Samsung Electronics Co., Ltd. for granting a leave of absence from Seoul. We thank the MGC Company of Japan for a gift of the compound. We also thank Nissan Chemical Company of Japan for RN1175 (the aligning agent). 
[1] J. F. Li, X. Y. Wang, E. Kanagas, P. L. Taylor, C. Rosenblatt, Y. Suzuki, and P. E. Cladis, Phys. Rev. B 52, R13075 (1995).

[2] A. Fukuda, Y. Takanishi, T. Isozaki, K. Ishikawa, and H. Takezoe, J. Mater. Chem. 4, 997 (1994).

[3] A. D. L. Chandani, T. Hagiwara, Y. Suzuki, Y. Ouchi, H. Takezoe, and A. Fukuda, Jpn. J. Appl. Phys., Part 2 21, L729 (1988).

[4] Y. Yamada, N. Yamamoto, K. Mori, K. Nakamura, T. Hagiwara, Y. Suzuki, I. Kawamura, H. Orihara, and Y. Ishibashi, Jpn. J. Appl. Phys., Part 2 29, 1757 (1990).

[5] N. Yamamoto, Y. Yamada, K. Mori, K. Nakamura, H. Orihara, Y. Ishibashi, Y. Suzuki, Y. Negi, and I. Kawamura, Jpn. J. Appl. Phys., Part 2 30, 2380 (1991).

[6] R. Beccherelli, N. J. Mottram, and S. J. Elston, Eur. Phys. J.: Appl. Phys. 9, 247 (2000).

[7] T. Qian and P. L. Taylor, Phys. Rev. E 60, 2978 (1999).

[8] L. A. Parry-Jones and S. J. Elston, Appl. Phys. Lett. 79, 2097 (2001).

[9] H. Orihara and Y. Ishibashi, Jpn. J. Appl. Phys., Part 2 29,
L115 (1990).

[10] M. Johno, K. Itoh, J. Lee, Y. Ouchi, H. Takezoe, A. Fukuda, and T. Kitazume, Jpn. J. Appl. Phys., Part 2 29, L107 (1990).

[11] E. Magyari, Phys. Rev. B 29, 7082 (1984).

[12] Lin Lei, Shu Changqing, Shen Juelian, P. M. Lam, and Huang Yun, Phys. Rev. Lett. 49, 1335 (1982).

[13] N. A. Clark and S. T. Largerwall, Appl. Phys. Lett. 36, 899 (1980).

[14] X. Y. Wang and P. L. Taylor, Phys. Rev. Lett. 76, 640 (1996).

[15] L. A. Parry-Jones and S. J. Elston, Phys. Rev. E 63, 050701(R) (2001).

[16] M. Johno, A. D. L. Chandani, Y. Ouchi, H. Takezoe, A. Fukuda, M. Ichihashi, and K. Furukawa, Jpn. J. Appl. Phys., Part 2 28, L119 (1989).

[17] J. K. Song, A. D. L. Chandani, O. E. Panarina, A. Fukuda, J. K. Vij, V. Goertz, and J. W. Goodby, Ferroelectrics 344, 41 (2006).

[18] N. M. Shtykov, A. D. L. Chandani, A. V. Emelyanenko, A. Fukuda, and J. K. Vij, Phys. Rev. E 71, 021711 (2005). 